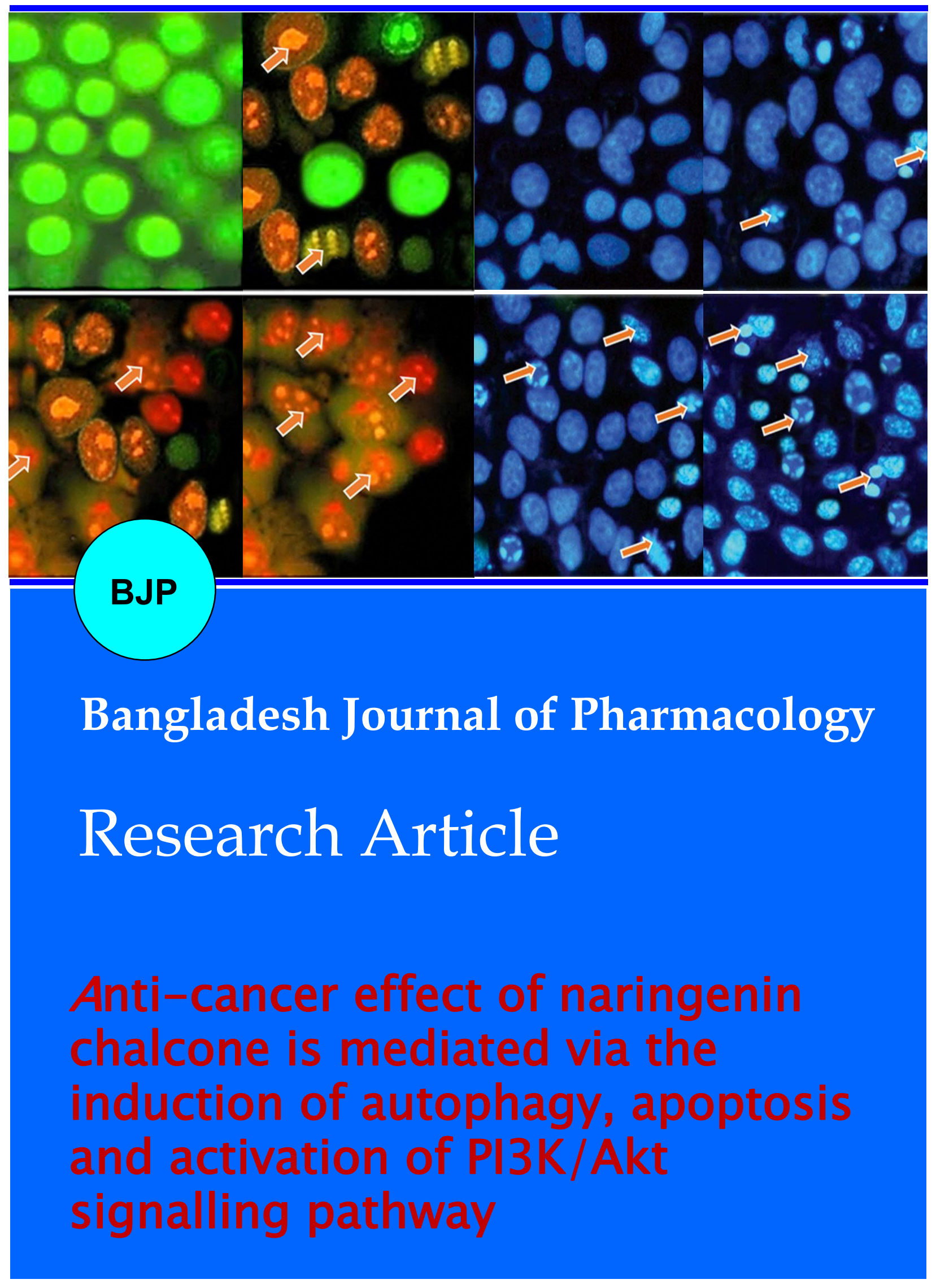


Abstracted/indexed in Academic Search Complete, Asia Journals Online, Bangladesh Journals Online, Biological Abstracts, BIOSIS Previews, CAB Abstracts, Current Abstracts, EMBASE/Excerpta Medica, Google Scholar, HINARI (WHO), International Pharmaceutical Abstracts, Open J-gate, Science Citation Index Expanded, SCOPUS and Social Sciences Citation Index

\title{
Anti-cancer effect of naringenin chalcone is mediated via the induction of autophagy, apoptosis and activation of PI3K/Akt signalling pathway
}

\author{
Shuai Zhang',2, Zong-Fei Jiang², Qiang Pan², Chun-Yu Song² and Wen-Hua Zhang'
}

${ }^{1}$ Department of Neurosurgery, Qilu Hospital, Shandong University, Jinan City, Shandong 250-012, China; ${ }^{2}$ Department of Neurosurgery, People's Hospital of Laiwu City, Laiwu City, Shandong 271-100, China.

\begin{tabular}{|c|c|}
\hline Article Info & \\
\hline Received: & 27 April 2016 \\
\hline Accepted: & 3 June 2016 \\
\hline Available Online: & 12 July 2016 \\
\hline DOI: $10.3329 /$ bjp.v & 27518 \\
\hline $\begin{array}{l}\text { Cite this article: } \\
\text { Zhang S, Jiang ZF } \\
\text { Zhang WH. An } \\
\text { naringenin chalco } \\
\text { the induction of au } \\
\text { and activation of } \mathrm{F} \\
\text { pathway. Banglac } \\
\text { 2016; 11: 684-690. }\end{array}$ & $\begin{array}{l}\text { Q, Song CY, } \\
\text { cer effect of } \\
\text { mediated via } \\
\text { agy, apoptosis } \\
\text { Akt signalling } \\
\text { J Pharmacol. }\end{array}$ \\
\hline
\end{tabular}

\begin{abstract}
The aim of the current study was to investigate the in vitro and in vivo antitumor effects of naringenin chalcone in U87MG human glioblastoma cells and in xenograft mice model. The effect of naringenin chalcone on apoptosis induction was assessed by fluorescence microscopy using acridine orange/ ethidium bromide and Hoechst 33342. Effect of the compound on PI3K/Akt signalling proteins was assessed by Western blot assay. Naringenin chalcone induced dose-dependent as well as time-dependent cytotoxic effects in these cells. Transmission electron microscopy showed that naringenin chalcone induced the formation of autophagic vacuoles. The number and size of these autophagic vacuoles increased with increasing dose of naringenin chalcone. It also led to the activation of both phosphorylated as well as nonphosphorylated PI3K and Akt proteins. In vivo results showed that both tumor volume and tumor weight were lesser in naringenin chalcone-treated groups with its different doses than in vehicle control group.
\end{abstract}

\section{Introduction}

Malignant gliomas are the primary brain cancers accounting for about $80 \%$ of the all cancers targeting central nervous system. Human glioblastoma continues to challenge all current therapeutic approaches. The standard treatment protocol for glioblastoma involves surgical resection followed by chemotherapy and radiotherapy. The median survival period for glioma patients is only 12-14 months despite latest advances in the treatment of malignant gliomas such as the use of novel chemotherapeutic agents like temozolomide or the antibody anti-angiogenic therapy (Stupp et al., 2005; Gerstner et al., 2007). The incompetence of many chemotherapeutic drugs to cross the blood-brain barrier is the principal reason of the limitations of chemotherapy in the treatment of human gliomas. This is the reason that there are very few active drugs available for its treatment. As such there is a pressing need for the design and development of novel and promising anti-glioma chemotherapeutic agents (Das et al., 2010).

Flavonoids are the most important and largest class of plant phytochemicals present in a variety of fruits, vegetables and beverages of plant origin. Flavonoids have been reported to exhibit anti-oxidant, anti-cancer and anti-mutagenic activities. These phytochemicals are believed to have a significant protective role in several chronic diseases including malignant tumors. This also explains the well-known relations between decreased risk of numerous cancers and high consumption of fruits and vegetables containing flavonoids (Ahmad et al., 2000; Brown et al., 1998; Chung et al., 2001; Ramos, 2008; Formica and Regelson, 1995).

Chalcone structure comprises of an open-chain flavonoid with two aromatic rings connected by a 3carbon fragment bearing $\alpha, \beta$-unsaturated carbonyl 
motif. They are isolated from the plants and are believed to be the synthons of natural products like flavonoids and isoflavonoids (Liu et al., 2015).

Naringenin chalcone also belongs to flavonoid class of phytochemicals and to the best of our knowledge, there are no such reports on the anti-glioma activity of naringenin chalcone reported in the published literature. So the aim of the present investigation was to study the anti-tumor potential of naringenin chalcone in U87MG human glioblastoma cells and in xenograft mice model along with evaluating its effects on apoptosis, autophagy and PI3K/Akt pathway.

\section{Materials and Methods}

\section{Chemicals and other reagents}

Naringenin chalcone (purity $>98 \%$; as determined by high performance liquid chromatography), 3-(4,5dimethyl-2-thiazolyl)-2,5-diphenyl-2H-tetrazolium bromide (MTT) were obtained from Sigma-Aldrich Chemical Co. (USA). Acridine orange/ethidium bromide (AO/ETBR) and Hoechst 33342 were purchased from Wuhan Boster Biological Technology Ltd. (China). Dulbecco's modified Eagle's medium, fetal bovine serum, penicillin, and streptomycin were purchased from Tianjin Hao Yang Biological Manufacture Co., Ltd. (China). Phosphorylated p-PI3K, PI3K, p-Akt and Akt were procured from Cell Signalling Technology, USA, while as X-ray film for chemiluminescent system was obtained from Fuji photo film, Japan. All other chemicals and solvents used were procured from Merck and Sigma-Aldrich.

\section{Cell line and cell culture conditions}

U87MG human glioblastoma cells were obtained from American Type Culture Collections and maintained in Dulbecco's Modified Eagle's Medium supplemented with $10 \%$ fetal bovine serum and $100 \mathrm{U} / \mathrm{mL}$ penicillin and $100 \mu \mathrm{g} / \mathrm{mL}$ streptomycin at $37^{\circ} \mathrm{C}$. Cells were cultured in $\mathrm{CO}_{2}$ incubator (New Brunswick, Galaxy $170 \mathrm{R}$, eppendroff) with an internal atmosphere of $95 \%$ air and $5 \% \mathrm{CO}_{2}$ gas and the cell lines were maintained at $37^{\circ} \mathrm{C}$.

\section{Cell proliferation assay (Video Clip)}

The cytotoxic effects induced by naringenin chalcone in U87MG human glioblastoma cells were evaluated by MTT cell cytotoxicity assay. In brief, U87MG cells at a density of $2 \times 10^{5}$ cells/well were seeded on a 96-well plates. After 24 hours, naringenin chalcone dissolved in dimethyl sulfoxide at various concentrations $(0,2.5,5$, $10,50,75$ and $100 \mu \mathrm{M})$ was added to the cells and incubated for 24 and 48 hours time intervals. The plates containing the treated cells were then treated with 10 $\mu \mathrm{L}$ MTT solution in PBS for 2 hours. The formazan crystals then formed were dissolved in dimethyl sulfoxide and the absorbance was measured on a microplate reader. The effects of naringenin chalcone on cell cytotoxicity were shown as an inhibition ratio (I\%).

\section{Fluorescence microscopic study of apoptosis}

Acridine orange/ethidium bromide double dye assay

The apoptotic effect of naringenin chalcone on the U87MG human glioblastoma cells was determined by fluorescence microscopy using acridine orange/ ethidium bromide double dye assay. In brief, U87MG cells were seeded in 6-well plates at a density of $2 \times 10^{5}$ cells/well and then treated with different concentrations $(0,10,75$ and $100 \mu \mathrm{M})$ of naringenin chalcone for 48 hours. Then, the untreated and treated cells were incubated with acridine orange $(10 \mu \mathrm{g} / \mathrm{mL})$ and ethidium bromide $(10 \mu \mathrm{g} / \mathrm{mL})$ for 2 hours and then the apoptotic/necrotic cell death was visualized using a fluorescent microscope (400x magnification, Nikon, Japan).

\section{Hoechst 33342 staining}

The fact that naringenin chalcone does induce apoptotic effects in U87MG human glioblastoma cells, Hoechst 33342 staining along with fluorescence microscope was used for further confirmation. In brief, U87MG cells were seeded at a density of $2 \times 10^{5}$ cells/well in a 6-well plates and then treated with different concentrations $(0$, 10,75 and $100 \mu \mathrm{M}$ ) of naringenin chalcone for 48 hours. After incubation for 48 hours, the cells were fixed with $3.5 \%$ formaldehyde for $30 \mathrm{~min}$ and after that washed with PBS three times. After washing, a solution of Hoechst 33342 staining dye was added to the cells and then after $20 \mathrm{~min}$ the cells were visualized under a fluorescent microscope (200x magnification, Nikon, Japan) for apoptotic morphological changes (Zhang et al., 2015).

\section{Autophagy evaluation of U87MG human glioblastoma cells by Transmission Electron Microscopy}

U87MG human glioblastoma cells $\left(2 \times 10^{6}\right.$ cells/well $)$ were seeded in three flasks. The cells were treated with increasing doses $(0,10,75$ and $100 \mu \mathrm{M})$ of naringenin chalcone for 48 hours and the cells were harvested and washed with PBS three times. Afterwards, 2.0\% glutaraldehyde was added for microtome sectioning using ultramicrotome (JEOL Co., Japan). TEM analysis was performed using a Transmission Electron Microscope (JEM-4000; JEOL Co., Japan). Autophagy evaluation was done by measuring the formation of vacuoles and their abundance in drug-treated cells.

\section{Apoptosis quantification using annexin V-FITC flow cytometric assay}

Annexin V-FITC assay (annexin V-FITC apoptosis detection kit) was used to quantify the extent of apoptosis induced by naringenin chalcone in U87MG human glioblastoma cells (Rasul et al., 2014). In brief, 
U87MG cells at a density of $2 \times 10^{5}$ cells/mL were seeded and then subjected to the treatment of naringenin chalcone at increasing doses $(0,10,75$ and $100 \mu \mathrm{M})$. Subsequently the cells were incubated for 48 hours, washed with PBS and then stained with propidium iodide and annexin V-FITC according to manufacturer instructions. The cells were analyzed by flow cytometry using FACS Calibur instrument (BD Biosciences, USA) equipped with Cell Quest 3.3 software.

\section{Western blot}

Using Western blot assay, the effect of naringenin chalcone on the PI3K/Akt signalling pathway was evaluated. In brief, U87MG human glioblastoma cells were firstly starved in serum-free ECGM for 4 hours and were pre-treated without or with naringenin chalcone $(0,10,75$ and $100 \mu \mathrm{M})$ for $40 \mathrm{~min}$. Following treatment, the cells were harvested and lysed and then centrifuged. The protein contents of the supernatants were calculated by the bicinchoninic acid method using a multimode microplate spectrophotometer. About 60$80 \mu \mathrm{g}$ of cellular protein from each sample was applied to $8-10 \%$ SDS-polyacrylamide gels and probed with specific antibodies followed by exposure to horseradish peroxidase-conjugated goat anti-mouse antibodies.

\section{Xenograft tumor model in nude mice}

The anti-tumor effects of naringenin chalcone were examined using a nude mouse model. Male Balb/c nude mice (8-weeks old) were purchased from Shanghai Laboratory Animal Center (China), and all mice were maintained with water and food ad libitum in a pathogen free environment with a 12 hours light and 12 hours dark cycle in an animal care facility. The U87MG human glioblastoma cells ( 1 × $10^{6}$ cells/mouse) were subcutaneously injected into the right rear flank of each mouse (6-8 mice per group) to produce tumors in mice. After tumor development, the mice were divided into 5 groups, viz., vehicle control group, naringenin chalcone-treated group with $5 \mathrm{mg} / \mathrm{kg}$ dose, naringenin chalcone-treated group with $20 \mathrm{mg} / \mathrm{kg}$ dose, naringenin chalcone-treated group with $40 \mathrm{mg} / \mathrm{kg}$ dose, naringenin chalcone-treated group with $80 \mathrm{mg} /$ $\mathrm{kg}$ dose. The mice were sacrificed after 24 days and the tumor weight and volume of each mouse were evaluated. Tumor length and width were measured using a caliper and the tumor volume was calculated using the formula:

Tumor volume $=$ length $\times$ width $\times 0.5$ width

\section{Statistical analysis}

The results are expressed as mean \pm SD of three independent experiments. Differences between the control and treatment groups were examined using the Student's t-test with SPSS 17.0 software. A p value $<0.05$ was considered to be statistically significant.

\section{Results}

Anti-tumor activity against U87MG human glioblastoma cells

It can be seen that with increasing concentrations of the compound, the cytotoxicity effect first showed slow increase but at concentrations above $50 \mu \mathrm{M}$, the cytotoxic effect showed a sharp increase. Further, the cytotoxicity pattern also seemed to follow incubation intervals to which these cells were subjected. The cytotoxicity effect also showed strong time-dependence and the effect increased in a time-dependent manner (Figure 1).

\section{Significant apoptosis in U87MG human glioblastoma cells}

Results indicate that naringenin chalcone induced significant morphological changes in these cancer cells and these morphological changes were reminiscent of apoptosis. The morphological changes included cell shrinkage and apoptotic body formation. Untreated cells showed green fluorescence indicating no apoptosis. On treatment with naringenin chalcone at increasing doses led to the appearance of red/orange fluorescence indicating onset of apoptosis (Figure 2A).

After this, Hoechst 33342 staining also indicated signs of apoptosis in these cells after treatment with naringenin chalcone at various concentrations. Figure 2B revealed the apoptotic effects induced by naringenin chalcone in U87MG human glioblastoma cells at increasing concentrations. As compared to control cells which exhibited normal cell morphology, naringenin chalcone-treated cells showed significant levels of apoptosis characterized by chromatin condensation, DNA fragmentation, membrane blebbing. As compared to control cells, naringenin chalcone treated cells showed bright fluorescence resulting from cleaved DNA.

\section{Autophagy in U87MG human glioblastoma cells}

The results obtained from Transmission Electron Microscopy indicated that naringenin chalcone treatment to U87MG human glioblastoma cells resulted in the appearance of autophagic vacuoles (Figure 3) and further, the number and size of these autophagic vacuoles increased with increasing doses of naringenin chalcone.

\section{Activated phosphoinositide 3-kinase (PI3K/Akt) signalling pathway}

The PI3K/AKT signaling pathway also controls a diversity of cellular proliferation and survival processes. The results which are shown in Figure 4 revealed that the compound led to the up-regulation of the protein expressions of all PI3K related proteins. It led to the activation of both phosphorylated as well as non-phosphorylated PI3K and Akt proteins. The un- 


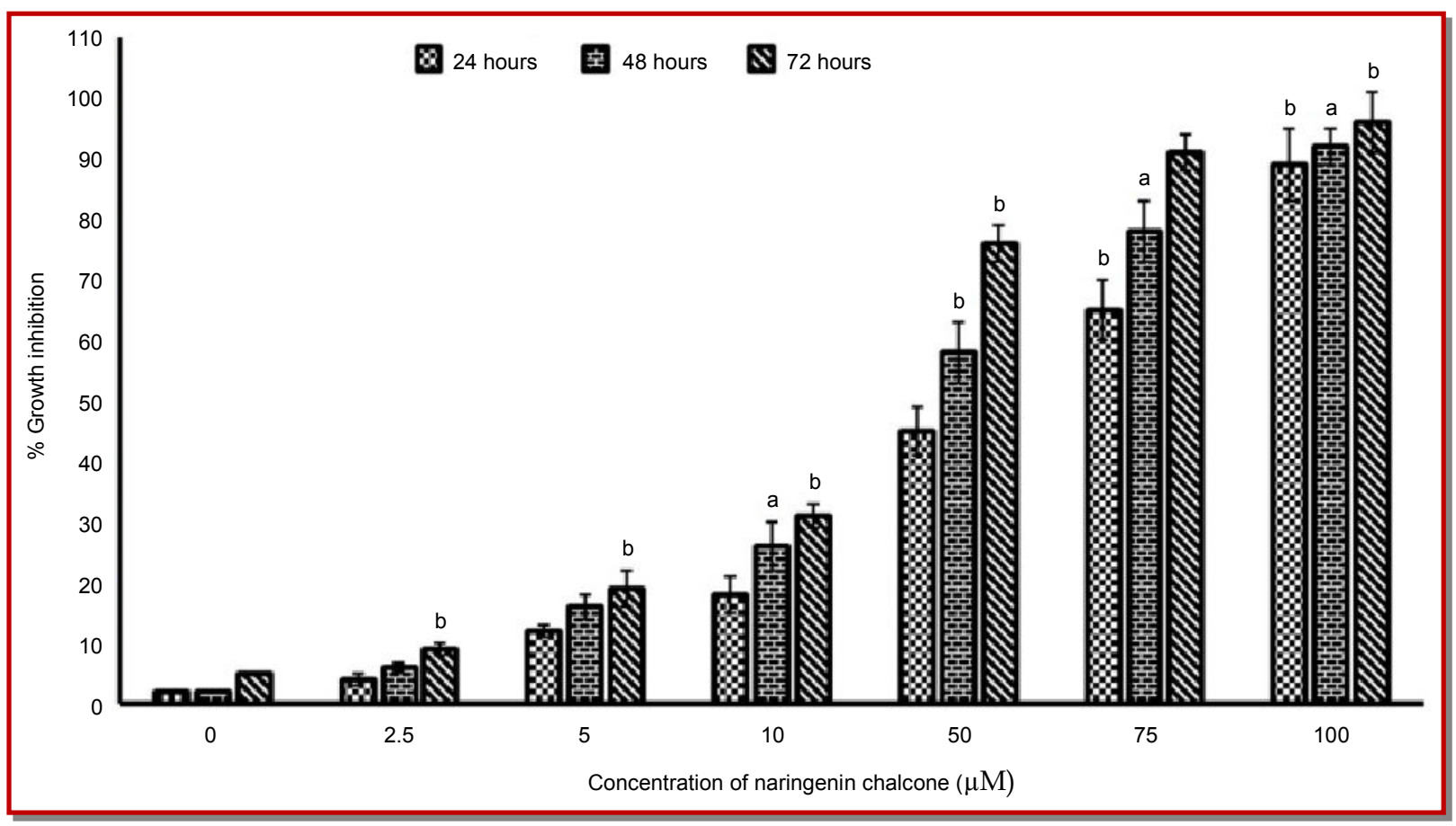

Figure 1: Effect of naringenin chalcone on the cell viability of U87MG human glioblastoma cells. The cells were treated with different doses of the drug and at three different time intervals. Data are shown as the mean \pm SD of three independent experiments. ${ }^{\mathrm{a}} \mathrm{p}<0.05$, b $\mathrm{p}<0.01$, vs $0 \mu \mathrm{M}$ (control)
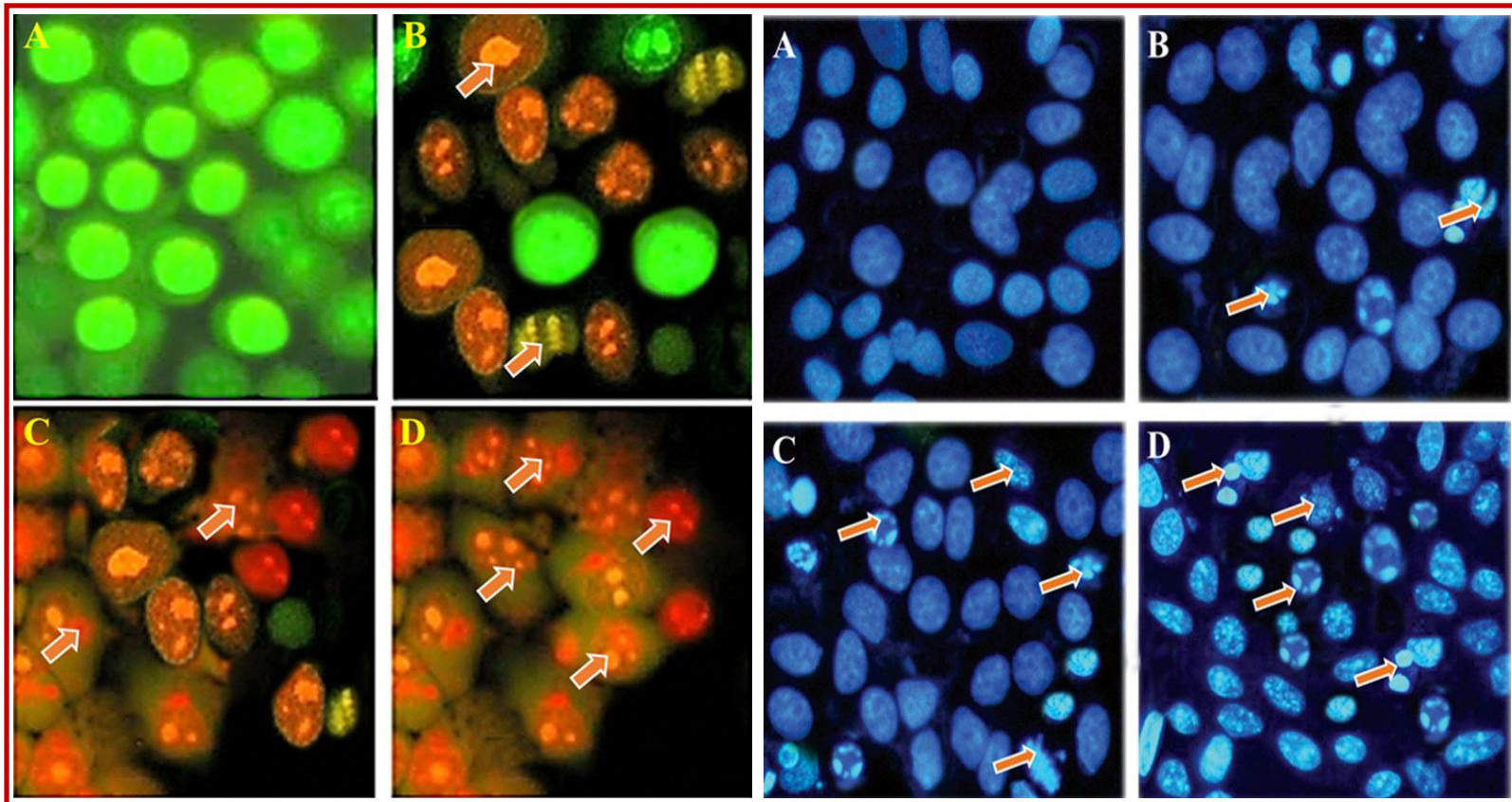

Stained with acridine orange/ethidium bromide

Stained with Hoechst 33342

Figure 2: Left two columns: Apoptosis induced by naringenin chalcone in U87MG human glioblastoma cells after the cells were treated with naringenin chalcone. The cells were treated with $0,10,50$ and $100 \mu \mathrm{M}$ of drug, stained with acridine orange/ethidium bromide, then observed under fluorescence microscope at 400x magnification. Red and orange fluorescence indicates cells undergoing apoptosis. Right two columns: Apoptosis induced by naringenin chalcone in U87MG human glioblastoma cells after the cells were treated with naringenin chalcone. The cells were treated with $0,10,50$ and $100 \mu \mathrm{M}$ of drug, stained with Hoechst 33342 , then observed under fluorescence microscope at 200x magnification. Arrows indicates cells undergoing apoptosis (bright fluorescence) 


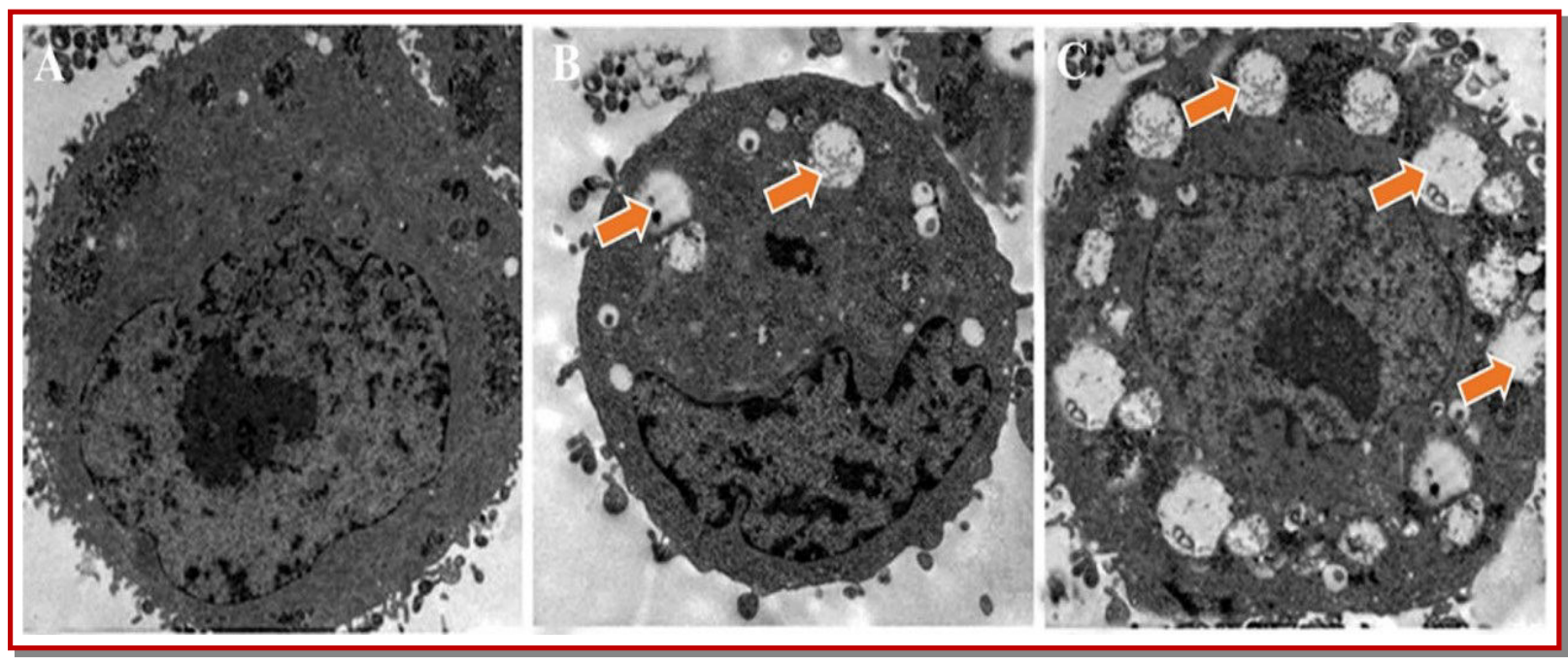

Figure 3: Evaluation of autophagy-induction by naringenin chalcone in U87MG human glioblastoma cells. The cells were treated with $0 \mu \mathrm{M}(\mathrm{A}), 50 \mu \mathrm{M}(\mathrm{B})$ and $100 \mu \mathrm{M}(\mathrm{C})$ dose of naringenin chalcone for 48 hours and then analyzed by Transmission Electron Microscopy. Arrows indicate autophagic vacuoles which were seen only in drug-treated cells

treated control group showed lower levels of protein expressions.

\section{In vivo anti-tumor activity}

It was observed that the rate of tumor development in nude mice was 100 percent. The tumor weight and tumor volume of control and naringenin chalconetreated groups $(5,20,40,80 \mathrm{mg} / \mathrm{kg}$ dose) was measured at 3 days intervals. The results indicated that the tumor weight and tumor volume in nude mice decreased

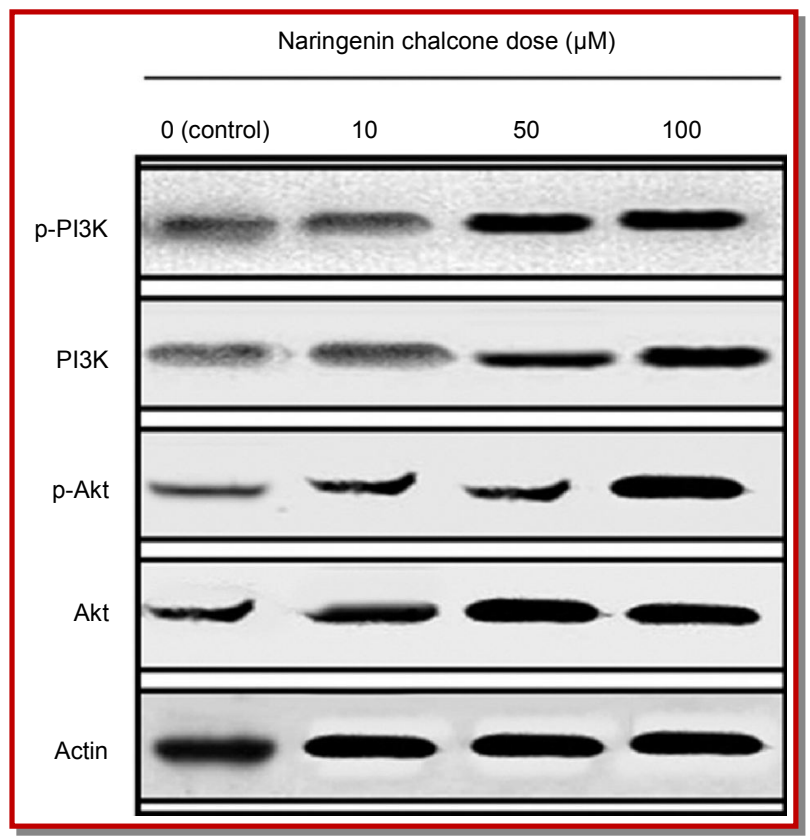

Figure 4: Naringenin chalcone targets PI3K/Akt signalling pathway in U87MG human glioblastoma cells. The cells were treated with 0, 10, 50 and $100 \mu \mathrm{M}$ and the protein expressions were evaluated by Western blot. The treatment led to upregulation of PI3K/Akt proteins considerably in the naringenin chalcone-treated group with $100 \mathrm{mg} / \mathrm{kg}$ dose in contrast to the control group. At the end of the experiment, both tumor volume and tumor weight was very less in naringenin chalconetreated groups with its different doses (Figure 5).

\section{Discussion}

In the current study, the in vitro and in vivo anti-tumor effects of naringenin chalcone were evaluated against U87MG human glioblastoma cells using MTT assay. In addition, the apoptotic effects of this compound on these cells were evaluated by fluorescence microscopy using acridine orange/ethidium bromide and Hoechst 33342 staining dyes. Further, the effect of naringenin chalcone on the autophagic induction in U87MG cells was demonstrated by transmission electron microscopy. Western blot assay was used to study the relationship between naringenin chalcone dose and the protein expressions of PI3K/Akt proteins. MTT results showed that naringenin chalcone not only showed dose -dependent but also time-dependent cytotoxicity towards U87MG human glioblastoma cells. Fluorescence microscopy revealed that naringenin chalcone induced potent apoptotic effects with the drug treated cells showing red/orange fluorescent patterns. Hoechst 33342 staining also showed similar results indicating that higher doses of the drug led to bright fluorescence indicating chromatin condensation and DNA fragmentation. The process of apoptosis is characterized by certain morphological changes including cell shrinkage, membrane blebbing and fragmentation into membrane-bound apoptotic bodies (Kerr et al., 1972; Saraste, 1999). Using transmission electron microscopy, the effect of naringenin chalcone on autophagy was evaluated. Naringenin chalcone 

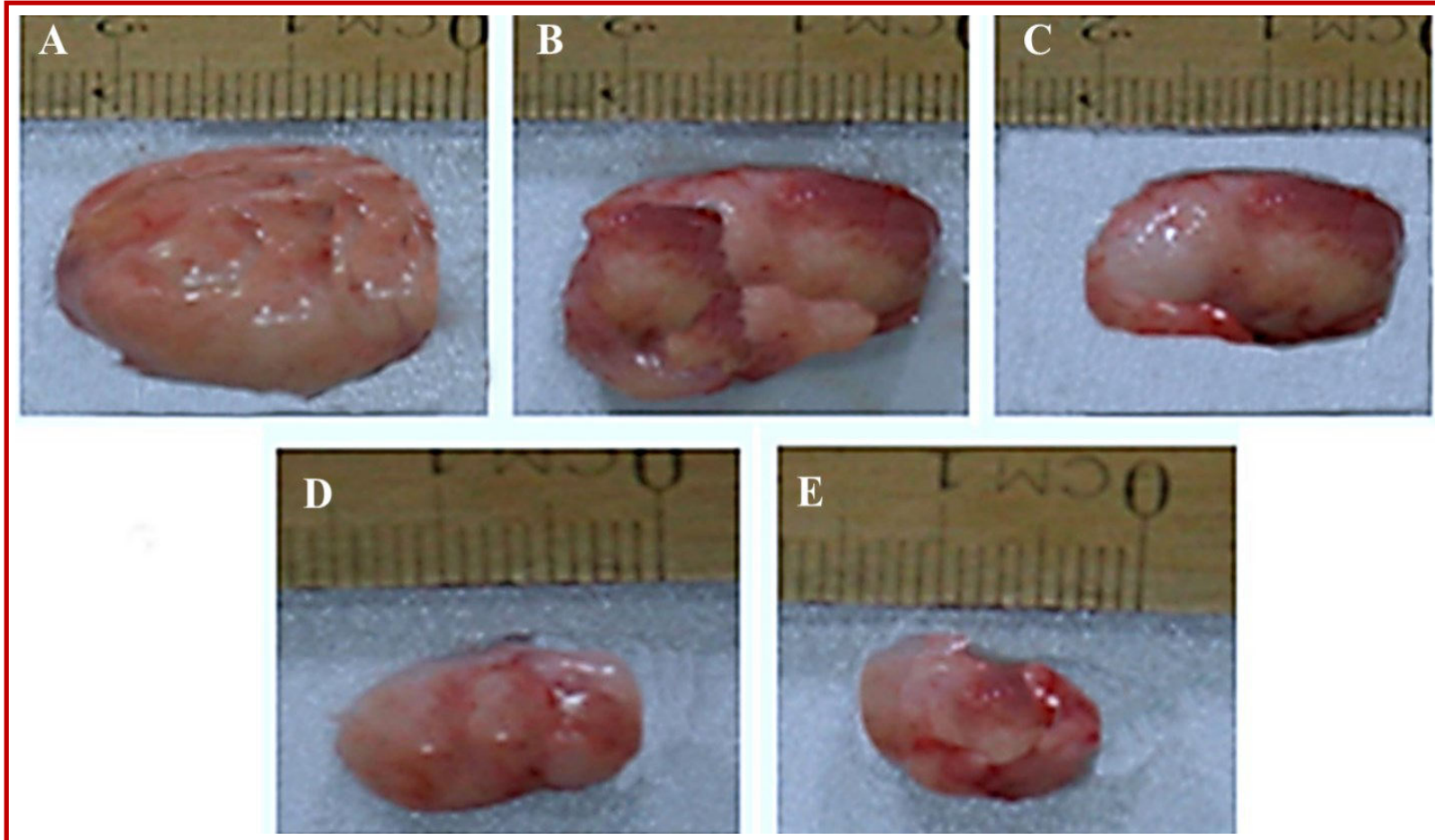

Figure 5: In vivo anti-cancer activity of naringenin chalcone on U87MG human glioblastoma cells in xenograft mice model. Cancerous Xenografts from U87MG human glioblastoma cells were incorporated in nude mice. The mice were treated with either vehicle control or naringenin chalcone for 8 days. A, shows vehicle control group, B, shows naringenin chalcone-treated group with 5 $\mathrm{mg} / \mathrm{kg}$ dose, C, naringenin chalcone-treated group with $20 \mathrm{mg} / \mathrm{kg}$ dose, $\mathrm{D}$, naringenin chalcone-treated group with $40 \mathrm{mg} / \mathrm{kg}$ dose, E, naringenin chalcone-treated group with $80 \mathrm{mg} / \mathrm{kg}$ dose

treatment to U87MG human glioblastoma cells resulted in the appearance of autophagic vacuoles and further, the number and size of these autophagic vacuoles increased with increasing dose of naringenin chalcone. Autophagy is intimately linked with tumors and plays a significant role in human tumor suppression, so inducing autophagy is a prospective therapeutic strategy in adjuvant chemotherapy (Zhang et al., 2011). Finally, the effect of naringenin chalcone on the PI3K/ Akt signalling pathway was demonstrated using Western blot assay. Naringenin chalcone led to the activation of both phosphorylated as well as nonphosphorylated PI3K and Akt proteins. The PI3K/Akt signaling pathway has been reported to play key roles in the occurrence of various malignant tumors (Osaki and Oshimura, 2004).

\section{Conclusion}

Naringenin chalcone exhibits anti-tumor activity in U87MG human glioblastoma cells and in xenograft mice model by inducing apoptosis, autophagy and activating $\mathrm{PI} 3 \mathrm{~K} /$ Akt pathway.

\section{Ethical Issue}

All of the animal experiments were conducted with the approval of the Animal Experimentation Ethics Committee of the People's Hospital of Laiwu City, Laiwu City, Shandong 271100, China.

\section{Conflict of Interest}

The authors declare that there is no conflict of interest to reveal.

\section{References}

Ahmad N, Cheng P, Mukhtar H. Cell cycle dys-regulation by green tea polyphenol epigallocatechin-3-gallate. Biochem. Biophys Res Commun. 2000; 275: 328-34.

Brown A, Jolly P, Wei H. Genistein modulates glioblastoma cell proliferation and differentiation through induction of apoptosis and regulation of tyrosine kinase activity and Nmyc expression. Carcinogenesis 1998; 19: 991-97.

Chung LY, Cheung TC, Kong SK, Fung KP, Choy YM, Chan ZY, Kwok TT. Induction of apoptosis by green tea catechins 
in human prostate cancer DU145 cells. Life Sci. 2001; 68: 1207 -14 .

Das A, Naren LB, Swapan KR. Flavonoids activated caspases for apoptosis in human glioblastoma T98G and U87MG cells but not in human normal astrocytes. Cancer 2010; 116: 16476.

Formica JV, Regelson W. Review of the biology of quercetin and related bioflavonoids. Food Chem Toxicol. 1995; 33: 1061 -80 .

Gerstner ER, Duda DG, di Tomaso E, Sorensen G, Jain RK, Batchelor TT. Anti-angiogenic agents for the treatment of glioblastoma. Expert Opin Investig Drugs. 2007; 16: 18951908.

Liu JJ, Zhang L, Lou JM, Wu CY. Chalcone derivative, chana 1 induces inhibition of cell proliferation and prevents metastasis of pancreatic carcinoma. Adv Biomed Pharm. 2015; 2 : 115-19.

Kerr JFR, Wyllie AH, Currie AR. Apoptosis: A basic biological phenomenon with wide-ranging implications in tissue kinetics. Br J Cancer. 1972; 26: 239-57.

Louis DN, Ohgaki H, Wiestler OD, Cavenee WK, Burger PC, Jouvet A, Scheithauer BW, Kleihues P. The 2007 WHO classification of tumours of the central nervous system. Acta Neuropathol. 2007; 114: 97-109.
Osaki M, Oshimura M. PI3K-Akt pathway: Its functions and alterations in human cancer. Apoptosis 2004; 9: 667-76.

Ramos S. Cancer chemoprevention and chemotherapy: Dietary polyphenols and signaling pathways. Mol Nutr Food Res. 2008; 52: 507-26.

Rasul A, Shen X, Wang B, Liu B, Li X, Tang J. Tubeimoside-1 upregulates p21 expression and induces apoptosis and G2/ $M$ phase cell cycle arrest in human bladder cancer T24 cells. Bangladesh J Pharmacol. 2014; 9: 595-603.

Saraste A. Morphologic criteria and detection of apoptosis. Herz 1999; 24: 189-95.

Stupp R, Mason WP, van den Bent MJ, Weller M, Fisher B, Taphoorn MJ, Belanger K, Brandes AA, Marosi C, Bogdahn U, Curschmann J, Janzer RC, Ludwin SK, Gorlia T, Allgeier A, Lacombe D, Cairncross JG, Eisenhauer E, Mirimanoff RO. Radiotherapy plus concomitant and adjuvant temozolomide for glioblastoma. N Engl J Med. 2005; 352: 987-96.

Zhang J, Li Y, Chen X, Liu T, Chen Y, He W, Zhang Q, Liu S. Autophagy is involved in anti-cancer effects of matrine on SGC-7901 human gastric cancer cells. Oncol Rep. 2011; 26: $115-24$.

Zhang J, Wen G, Zhang L, Duan D, Ren Z. Sulphureuine B, a drimane type sesquiterpenoid isolated from Laetiporus sulphureus induces apoptosis in glioma cells. Bangladesh J Pharmacol. 2015; 10: 844-53. 


\section{Your feedback about this paper}

1. Number of times you have read this paper 0

2. Number of times you have seen the video clip 0

3. Quality of paper Click

4. Your comments

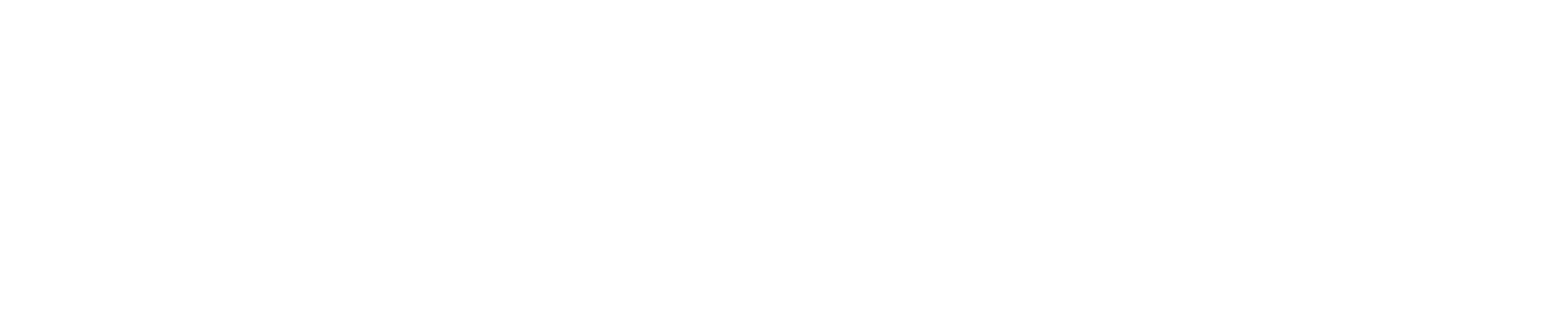

